\title{
UNA REGLA QUE NO FALL PARA EL CONTROL DE LA ACUÑACIÓN DE PACÍFICOS
}

\author{
R. Conde y Delgado de Molina
}

En su origen, la moneda no fue otra cosa que una cierta cantidad de oro, plata u otra materia de valor cuya cantidad estaba garantizada por el poder político, fuera cual fuera la función de este poder. A esto responde la clásica definición de san Isidoro: figura, pondus et metallum. Es decir, una cantidad determinada de un metal garantizada por una señal externa aceptada por cuantos deben hacer uso de ella.

Pero el poder no ponía gratuitamente su sello: de la diferencia entre el precio de coste y el valor de circulación de la moneda se derivaba un beneficio para la autoridad garantizadora, máxime cuando el bimetalismo permitió un verdadero agio sobre la moneda. Los reyes medievales, y con ellos cuantos tuvieron poder de acuñación, hicieron de la moneda, al tiempo que un signo de poder, una fuente de ingresos. Al control de estos ingresos va dirigido el de los gastos de acunación, que se traducian en la confección de una serie de libros «de monedero», "de fundidores», «de compras» de oro o plata, etc., que constituyen la documentación básica para el estudio de las acuñaciones'.

' De la variada documentación que el control de las acunaciones monetarias produce, queda reflejo en el trabajo de F. UDina MARToRELL, "La numis- 
A facilitar este control va dirigido el documento que presento, relativo a la acuñación de pacíficos en Cataluña. El pacífico, de creación de Pedro de Portugal en 1465, fue de ley de 20 quilates y curso de $18 \mathrm{ss}^{2}{ }^{2}$, y tuvo corta vida en cuanto que estuvo ligado a factores políticos. Juan II tras su victoria en la guerra civil catalana dejó pronto de acuñarlo. Durante los años 1465 y 1472 se acuñaron, según cuentas globales de las acuñaciones, un total de 145.727 pacíficos entre piezas de unidad, medios pacíficos y cuartos de pacífico o «quarterolas», de los cuales 17.213 lo fueron bajo Pedro de Portugal y 128.514 bajo Renato de Anjou, operaciones que supusieron un beneficio neto global de 15,974 ll. 17 ss. 11 d: ${ }^{3}$.

El texto, orientado al cálculo del beneficio de la operación, es de la misma mano que el Assumament universal de tot lo batiment fet per en Raffel Moliner, mestre de la secha de Barcelona', y que se refiere a los siete años en que se acuñó esta especie monetaria bajo los reyes intrusos. Tal vez formó en origen parte del cuadernillo mencionado o conjunto con él, pues al dorso de éste puede leerse: Assi ha algunes regles de aquest batiment de paciffichs, que pueden referirse a nuestro texto.

Su contenido puede articularse, y de hecho así to he hecho para facilitar su comentario, en doce capitulos convencionales cuya glosa constituye el objeto de este estudio, que viene a prolongar en la cronología los trabajos que Federico Udina Marto-

mática en los archivos», Numisma 150-155, 1978, pp. 593-612 en que recoge datos de los archivos Histórico Nacional, de Indias, de Simancas y de la Corona de Aragón.

${ }^{2}$ La creación y la acuñación del pacífico ha sido estudiada por BONET I Sisó, Les monedes catalanes, Barcelona 1909 vol. II, pp. 284 y ss. Botet, que exploró concienzudamente los registros de la Cancilleria real, no vio, como es sabido, los fondos del Maestre Racional. Halló en los de cancilleria las órdenes de acuñación de Pedro de Portugal y de Renato de Anjou, pero no pudo precisar la acuñación de pacificos en Palamós bajo Pedro y las cuatro acuñaciones de Renato.

${ }^{3}$ Vid. datos globales de la acunación en ACA. Real Patrimonio, Maestre Racional, 1992/3 (en adelante ACA.RP.MR.).

4 Titulo del texto reseñado en la nota anterior. 
rell publicó, a lo largo de su dilatada carrera como investigador, sobre monedas'.

El capítulo 1 contiene las normas para el control del metal amonedado, a partir, naturalmente, en éste y en los restantes puntos, de los datos facilitados por los libros realizados durante la acuñación.

El punto I.1 se anuncia como regla que no fall para computar la acuñación del oro llevado a la ceca. Establece la siguiente: oro fino $(+$ liga $)+$ sosteniment 0 refuerzo $=$ delliurances $o$ metal realmente amoneado + restos en granalla + restos de sisa$l l a+$ pérdidas o minves de fundición y coloración.

Para su aplicación, los puntos I.2 y I.4 establecen las relaciones entre el oro fino (teóricamente de 24 q.) y el oro aleado a 20 q. (ley del pacífico), desde 5 marcos hasta 1.000 marcos (1.2) y de 5 marcos a 5 granos (I.4), y concluye con la equivalencia universal de que 5 de fino equivalen a 6 de aleado. Los puntos I.3 y I.5, el sostenimiento o refuerzo de calidad de la moneda, fijado y ordenado por el rey, de una onza de plata de 0,666 (dos partes de plata y una de cobre) por cada 10 marcos de oro; el I. 3 de 10 marcos a 1.000 marcos y el I.5 de 10 marcos a 22,5 granos.

Como ejemplo práctico de la regla y del uso de las tablas anteriores usa, sin citarlos como tales, datos de la segunda acuñación de pacíficos de Renato, desarrollada entre 1468 y $1469^{6}$ en un balance hecho según la regla (I.1), balance en que se detectan pequeños errores de cálculo ( $4 \mathrm{oz} .13$ drs. $131 / 4$ grs. no jus-

5 Singularmente «Los alfonsinos de oro, primera acunación de este metal en la Corona de Aragón", Numisma 29, 1957; "Un aspecto de la evolución económica sarda en el s. xıv: la acuñación de monedan; CHCA, Cerdeña 1957; «La ceca de Barcelona en tiempos de Fernando de Antequera y de Alfonso el Magnánimo en relación con la situación económica de la ciudad", Numisma 34, 1958; «Una moneda inédita de Cervera», Numisma 6, 1953; «El movimiento económico del tiempo de Fernando de Antequera a través de sus acuก๊aciones», IV CHCA, 1955.

${ }^{6}$ ACA.RP.MR. 1992/3, fol, 3-4. 
tificados) y ligeras diferencias en los datos respecto a los aportados por la evaluación global.

El capíutlo II plantea la regla para el cálculo del beneficio de la operación en los siguientes términos (II.1):

a) Cálculo del número de pacíficos. Los acuñados realmente, sea en piezas de unidad o de sus divisores, más la valoración en pacíficos de los restos de granalla, etc. Metal justificado neto: 68 (talla del pacífico).

b) Valor en libras calculando el pacífico a 18 ss.: número de pacíficos $\times 18 \mathrm{ss}$.

c) Beneficio bruto en libras a 18 ss. pacífico: valor en libras - coste del oro fino.

d) Beneficio bruto en libras a 20 ss. pacífico: beneficio bruto a 18 ss. pacífico $\times 11 \div 10$.

e) Beneficio neto: beneficio bruto a 20 ss. pacífico - gastos de acuñación.

Aparece por primera vez en el texto una diferenciación entre pacíficos de 18 ss. y pacíficos de 20 ss., o entre lliures de fort, alias de 18 ss. paciffich y lliures de flaca, alias de 20 ss. paciffich. En la primera moneda se pagarán, en las cuentas reales, el oro fino, en tanto que en la segunda los gastos de acuñación y salarios de los trabajadores?. Confieso mi perplejidad ante este hecho. El pacífico se crea, y sale, a 18 ss. No he hallado en la bibliografía disponible rastros de mutación en el curso en el campo rebelde. En su campo, Juan II había cambiado el cur-

7 Vid., p.e. el detalle de las cuentas correspondientes a la acuñación de 1465-1466: Summa, dons, lo procehit del or, segons appar atras... paciffichs... los quals reduyts de 18 ss. paciffichs dels quals se deu pagar lo cost del or fi comprat per lo mestre, fan de moneda fort... Resta de moneda fort... les quals reduydes a moneda flaca de 20 ss. paciffich de que.s deven pagar messions e salaris de officials... (ACA.RP.MR. 1992/3, fol. $2 v^{\circ}$ ). En los mismos términos se expresan los balances parciales del periodo 1467-1472 (Ibid. fol. 5-5 $\mathrm{v}^{\circ}$ y 8 ) y general del periodo (Ibid. fol. $9-9 \mathrm{v}^{\circ}$ ). 
so de la moneda con anterioridad al 6 de septiembre de 1472 , poniendo los pacíficos en 20 ss., disposición que es anulada en esta fecha ${ }^{8}$ y renovada en $1476^{\circ}$. Si las cuentas son para Juan II, no pueden ser anteriores a la caída de Barcelona (octubre de 1472) ni anteriores al nuevo curso de 1476. ¿Significa esto que en esta fecha aún no se habian pagado las messions de la acuñación y que Juan II había asumido dichos gastos? Si son para el bando contrario, ¿en virtud de qué evalúa el pacífico unas veces a un curso y otras a otro, y en virtud de qué se pagan unos gastos en una moneda y otros en otra?

Otra duda plantea el hecho de la no valoración de la liga, que era de plata pura (1.2), ni del sosteniment, que tenía 0,66 milésimas de fino. ¿Debe considerarse incluido su valor en los gastos generales? No son, desde luego, cantidades despreciables. Sin ir más lejos, la liga puesta por el maestre de la ceca en la segunda acuñación de Renato, cuyos datos son usados por el autor del texto, supuso 61 marcos 1 onza 7 d. y $3 / 4$ de grano de plata marcat ... a 11 d. 12 grans y 6 marcos 3 onzas 9 dineros y 16 granos de plata de 0,66 milésimas para el sosteniment ${ }^{10}$.

Y atención al punto d) del citado capítulo II.1, puesto que no es válida la equivalencia, como más adelante se comenta largamente. Baste decir aquí que la regla para convertir libras a 18 ss. pacífico en libras a 20 ss. pacífico consiste en dividir las primeras por 18 y multiplicarlas por 200 , dicho en otros términos, el valor de las libras a $18 \mathrm{ss}$. pacífico es el mismo valor en libras que el número de pacíficos que entran en las citadas libras de 18 ss. pacifico ${ }^{11}$.

${ }^{8}$ Botet I Siso, op. cit., p. 296 (ACA. Cancilleria, reg. 3456, fol. 114 vำ115).

9 Botet i Siso, op. cit., p. 298.

10 ACA.RP.MR. 1992/3, fol. 3. El cobre del sosteniment montó 3 marcos 1 onza 16 dineros y $201 / 2$ granos, y 67 marcos 2 onzas 13 dineros y 6 granos la liga "que llevaba en si el oro" y que se incorpora a la aleación.

11 El error se repite a lo largo de todo el texto, aunque por fin es salvado. En las cuentas reales se reduce correctamente. Concretamente, en la evaluación global de la acuñación de la moneda, el beneficio bruto, en pacíficos de 18 ss., asciende a 17.790 II. 1 ss. 11 1/4 d. (con un ligero error de casi 1 sueldo en más: la cantidad exacta es 17.790 II. 11 3/4 d.) equivalentes a 19.766 Il. 15 ss. 5 d. en pacíficos de 20 ss. 
El párrafo II.2 propone un ejemplo práctico convencional a partir de la acuñación de 764 marcos 4 onzas y 15 dineros, ejemplo que queda interrumpido a partir de la resta de 40.000 libras (cantidad probablemente incompleta), precio del oro fino comprado por la ceca.

El capítulo III resume las bases definitorias de la acuñación. Renato de Anjou no varió en nada el pacífico de su antecesor. La orden de acuñación, de $30^{12}$ de septiembre de 1467 , fijaba la ley en 20 quilates con una tolerancia de $1 / 4$ de quilate en más o menos, la talla de 68 en marco y el curso de 18 ss. La moneda tendría los mismos tipos que la de su antecesor pero iría, naturalmente, batida a su nombre: Renatus primus Dei gratia rex Aragonum ${ }^{13}$.

Con la misma fecha se renueva el premio de dos quilates de oro fino por marco ${ }^{14}$ a fin de atraer oro a la ceca, y que ya había creado su antecesor. Recordemos que estamos en una etapa de contracción general de acuñaciones de oro ${ }^{15}$, contracción a la que hay que añadir en Barcelona los problemas derivados de la guerra.

El premio de 2 quilates de oro suponía el pago de 96 ss. por marco de oro. Valorado éste a 57 11. 12 ss. (III.3), significaba un beneficio mínimo del $8,3 \%$, que podía llegar a índices incalculables según la calidad del oro vendido, puesto que el premio se paga sea cual sea la calidad del oro (III.5).

12 No del 13 como, por un lapsus sin importancia, interpretó Boter I Siso el tricesimo del documento.

13 Vide supra nota 2. El original ACA. Cancilleria, intrusos, reg. 45, fol. $4 v^{\circ}-5 v^{\circ}$.

14 Ibid. fol. 5-5 $v^{\circ}$.

is J. DAr, The question of monetary contraction in late Medieval Europe, en Nordisk Numismatisk Arsskrift 1981, pp. 12-19. La inflexión, tanto en oro como en plata (pp. 21 y ss.) es clara a partir de 1441-1450. 
Constituyen el capítulo IV las tablas para calcular el precio de compra del oro fino (IV.1) a partir del precio de mercado de 5711.12 ss. el marco, o 2 II. 8 ss. el quilate, desde el marco hasta el grano, y del enantatge o premio (IV.2) comentado, desde el valor de 2 quilates por marco hasta el de este mismo valor por grano (cantidad ésta que deja en blanco por despreciable: correspondería a $3 / 16$ de dinero). Estos cálculos son repetidos, simplificados en función de los divisores del marco, en los puntos IV. 3 y IV.4.

El punto IV.5 viene a repetir los cálculos del beneficio de la acuñación explicados en el capítulo II ya comentado, referido a la acuñación de un marco: $811.18 \mathrm{ss}$. $1 \mathrm{~d}$. de beneficio bruto en moneda fuerte 09 Il. 16 ss. 9 d. en moneda flaca, cantidad de la que deben deducirse los gastos de acuñación: 111.10 ss. de carbón, crisoles, fundir, etc. y 1 II. 2 ss. por conceptos que, por ilegibilidad del original, no pueden precisarse.

El capítulo V establece la relación entre el marco de oro fino, de 24 quilates, y el marco ya aleado a 20 quilates, para resolver la equivalencia entre una cantidad de oro fino escogida, creo, al azar, 31 marcos 7 onzas y esta misma cantidad aleada a 20 quilates (V.2), porque « 25 marcos (de 24 quilates) son 30 marcos de 20 quilates porque 5 son 6 \%, de acuerdo con las conclusiones ya expuestas en otro punto (I.4). ¿Por qué está tachado? Confieso no tener respuesta para ello.

El párrafo 3 del capítulo $V$ se refiere, según parece, al cálculo de los gastos generales, no sólo del proceso de fundición y acuñación, y precisa que la toca o patrón de la aleación es de 19 $3 / 4$ quilates, es decir, 1/4 quilate menos, cuarto de quilate que será de plata, no de liga de cobre (es decir, $1 \mathrm{oz}$. por 10 marcos, apenas un 0,0125 por ciento), pero que a efectos de valoración se calculará o computará como de oro en beneficio del rey. No he hallado tal disposición real. En la orden de acuñación, ya citada, se indica únicamente que la tolerancia será de $1 / 4$ de quilate, sin indicar ni precisar más. La «regla» se encuentra también 
cancelada con una nota al margen incomprensible: videretur ut melius, sin sentido a menos que esté incompleta.

$$
\text { *** }
$$

Integran el capítulo VI las tablas de equivalencias de marcos a piezas de pacíficos a partir de la talla de 68 en marco, primero descendiendo del marco al grano (en un alarde de cálculo inútil) (VI.1), y ascendiendo luego del marco a los 1.000 marcos (VI.2). A destacar, porque forma parte del sistema contable, la mención del valor del oro que no llega a constituir un pacífico, un medio pacífico o un cuarto de pacífico (por más que los 4 ss. $6 \mathrm{~d}$. que aparecen en uno de los asientos deberían constituir el valor de una cuarterola).

A destacar también un hecho. Se acuñaron medios y cuartos de pacíficos ${ }^{16}$ pero en ningún momento, ni en estas reglas teóricas ni en las cuentas efectivas, se hace mención de estos subdivisores. Las cantidades globales acuñadas, ya mencionadas, integran piezas de diverso valor, sin que podamos saber la proporción relativa de las mismas.

De agradecer es el memorial del capítulo VII, que recuerda la relación entre el marco, la onza, el dinero y el grano, primero de forma directa (VII.1), y luego expone la reducción de dineros a onzas (VII.2).

El capítulo VIII forma las tablas de valoración de los pacíficos en libras barcelonesas. Gran parte del mismo está cancelado por evidentes errores que veremos.

16 Botet I Siso, op. cit., pp. 307 y ss. y M. Crusaront, Numismática catalano-aragonesa, Madrid 1982, pp. 121 y $5 s$. 
Del punto VIII.1 sólo es exacto el segundo apartado, que establece que 100 pacíficos equivalen a $90 \mathrm{Il}$. El resto es erróneo: 100 pacíficos contados a 20 ss. son 100 Il., no 110 ; 10 (pacíficos) de (moneda) fort no equivalen a 1111 ., sino a 10; 90 pacíficos computados a $18 \mathrm{ss}$. no equivalen a $99 \mathrm{Il}$. computándolos a 20 ss, sino a 90 ll.; 10 pacíficos (computados a 20 ss.) no son 11 II., sino 10 , y por lo mismo, 100 pacíficos no son 110 Il., sino $100 \mathrm{ll}$.

El resto de los puntos es válido. El VIII.2 da la equivalencia de pacíficos en libras desde 100 hasta 1; el VIII.3 quiere dar la equivalencia inversa, de libras en pacíficos, aunque gráficamente yerra la presentación; el VIII. 4 continúa con la equivalencia anterior desde 200 hasta 5.000 pacíficos.

El punto VIII.5 puede considerarse apéndice del VIII.1. Es válido y debe leerse: $5 \mathrm{ss}$. de flaca, es decir, de pacífico calculado a 20 ss. (equivalentes a $5 / 20$ de pacífico), equivalen a 4 ss. 6 d. del pacífico calculado a 18 ss. (aritméticamente $5 / 20 \times 18$ ).

El capítulo IX quiso establecer las tablas de relación o equivalencia entre pacíficos, moneda fort y moneda flaca. Sólo es válida la parte central del punto IX.1, que establece la valoración del pacífico en libras de moneda fort y que repite cálculos ya vistos. A la derecha de estas equivalencias quiso escribir el autor la relativa a moneda flaca, repitiendo errores anteriores y posteriores. Tal vez se dio cuenta, pues deja prácticamente en blanco esta columna, y tuvo que darse cuenta de que si un pacifico vale $1 \mathrm{ll}$. de flaca, cálculo exacto, 2 pacíficos no podian valer la extraña cifra de 1 Il. 19 ss. $61 / 4$ d., sino, lisa y llanamente, 2 Il. A la izquierda, y prácticamente ilegibles, se entrevén las cifras en arábigo que corresponden a la equivalencia de pacífico en moneda flaca, $\tan$ equivocada como la anterior, y por ello tachada.

De este cálculo pretende el autor sacar una regla IX.2, de la cual sólo es válido el segundo apartado, que afirma que 100 pacificos valen 90 II., y son erróneos los restantes. 
De gran interés es la nota del punto IX.3, pues nos revela las fuentes del constante error en la reducción de los pacíficos a moneda flaca. Debe interpretarse: Nota que los 9 (ss. del pacífico de 18 ss.) son 10 (ss. del pacífico de 20 ss., porque 18 ss. son 20 ss.). Hasta aquí, cierto. El error viene a continuación: ... y 20 (ss.) de fort son 22 (ss.) de flaca... Falso porque, en realidad, equivalen, guardando las proporciones, a 22 ss. $4 / 18$ de sueldo $^{17}$ o $22 / 3$ d. Pero el considerar que si 18 vale 10,20 valdrán 22 , tiende una trampa al autor, pues, como en un punto posterior corrige, se desliza, sin sentirlo, de considerar que 1 pacifico vale 18 ss. y considerar que 1 pacífico vale 20 ss., a considerar que éstos 20 ss. son de moneda fort y que deben sumarse otra vez otros 2 ss. de diferencia entre la fort y la flaca. Curiosamente las tablas que siguen son válidas, pues parten de la equivalencia de que 18 ss. de fort valen 20 de flaca, equivalencia correcta.

El capítulo $\mathrm{X}$ pretende crear una nueva regla relativa al costo de la operación a partir de un punto básico: el oro comprado se paga en pacificos de 18 ss., la moneda fort, en tanto que los costes de fundición, acuñación, etc., en moneda flaca de 20 ss., extremo éste no precisado en el texto, pero claramente expresado en la contabilidad real de la operación.

La regla ordena:

a) Reducir a marcos el número de pacíficos acuñados a $68 \mathrm{el}$ marco.

b) Evaluar estos pacíficos a 18 ss. pieza.

c) Restar a esta cantidad el coste del oro fino comprado.

d) Reducir este resto, que será en moneda fort a moneda fla$c a$, presuponiendo que este resto son pacíficos, y aumentar asi 2 ss. por libra, alias por pacífico. Nuevamente comete el error comentado en el punto IX.3.

Como ejemplo calcula el beneficio de la acuñación de 10 marcos de oro:

$1718: 20: 20: X ; x=(20 \times 20): 18=400=18222 / 9 \mathrm{ss}$. 
a') Diez marcos de oro equivalen a 680 pacíficos.

$\left.b^{\prime}\right)$ Valor a 18 ss. pieza $\ldots \ldots \ldots \ldots \ldots \ldots \ldots \ldots, 612 \mathrm{II}$.

c') Coste del oro fino .................. $500 \mathrm{ll}$. Resto ........................... $112 \mathrm{ll}$.

Hasta aquí, el cálculo es correcto. Pero luego sigue aplicando el criterio erróneo, pues considera pacíficos de 20 ss., a las 112 II., a los cuales pacíficos, según él, hay que añadir otros 2 ss., lo cual significa, en la práctica, que añade 4 ss. por pacífico, aumentando su valor a 22 ss., en lugar de a 20 ss. La prueba es que continúa evaluando las $100 \mathrm{ll}$. (de pacíficos en moneda fort ) en $110 \mathrm{ll}$. de pacíficos en moneda flaca, por lo que las 112 II. se le convierten en 123 II. 4 ss., cuando, en realidad, el cálculo debería ser:

112 pacificos $\times 18$ ss. $\ldots \ldots \ldots \ldots \ldots \ldots .2016$ ss.

$112 \times 2$ ( 2 ss. por pacífico $) \ldots \ldots \ldots \ldots 224$ ss.,

total equivalente, pues, a 112 ll.; es decir, que, realmente, para pasar de moneda fort a flaca, o de pacíficos de moneda fort a pacíficos de moneda flaca, el número de unos equivale al número de libras de flaca, lo cual significa que el pacífico vale 1 libra, o sea, los 20 ss. tantas veces citados.

Por fortuna el autor se da por fin cuenta de su error: Aquesta regla es falsa, etc., para concluir notando que 1 pacífico de fort no valga de flaca 1 ll. 2 ss. (es decir, 22 ss.), más reduciendo el resto que queda pagado el oro, no se debe tomar tal cómputo ni reducir (de manera) que 1 (pacífico) de fort sean 22 ss. de flaca, sino que $18 \mathrm{ss}$. (1 pacífico de fort) son $20 \mathrm{ss}$. (de flaca, sumados los 2 ss. por pacífico).

El capítulo XI se refiere a cálculos de la liga del metal a amonedar.

El primer punto plantea una operación evidente:

Oro aleado a ley de pacífico - oro comprado = liga añadida puesto que el oro comprado tiene en sí ya liga. 
El punto XI.2 se refiere al sosteniment o refuerzo de la aleación añadido al oro ya aleado de 20 quilates:

Sosteniment + oro aleado a ley de 20 quilates $=$ oro aleado y reforzado,

que es el realmente acunado.

El punto XI. 3 vuelve a referirse a la liga planteando cálculos más directos sens fer assumament particular:

a) Oro aleado a ley de pacífico - oro comprado = liga.

b) Liga total - liga añadida en la ceca $=$ liga que tenía el oro al comprarse.

c) Oro comprado encamerat fino + liga originaria.

En resumen, utilizando datos de la segunda acuñación de Renato como en otras ocasiones, y prescindiendo, como hace en la segunda parte del capítulo, de dineros y granos, el cálculo es:

1. Oro fino comprado Liga originaria

Oro encamerat

2. Oro encamerat

Liga añadida en la ceca

Oro aleado a $20 \mathrm{q}$.

3. Oro aleado a $20 \mathrm{q}$. Refuerzo

Oro aleat e sostengut, realmente acuñado
$642 \mathrm{~m} .3 \mathrm{oz}$.

$67 \mathrm{~m} .2 \mathrm{oz}$.

$709 \mathrm{m.} 5 \mathrm{oz}$.

709 m. 5 oz.

$61 \mathrm{~m} .2 \mathrm{oz}$.

$770 \mathrm{~m} .7 \mathrm{oz}$.

$770 \mathrm{~m} .7 \mathrm{oz}$.

$\frac{9 \mathrm{~m} .5 \mathrm{oz} .}{780 \mathrm{~m} .4 \mathrm{oz} .}$

El capitulo XII y último me resulta incomprensible. Sin duda se refiere a la evaluación económica de la operación, pues principia con un Lo preu que costa..., pero no he logrado desentrañar su intención. 


\section{APÈNDIX}

Ihesus christus ${ }^{18}$

(de otra mano) De paciffichs

ACA, Real Patrimonio, sig ${ }^{\mathrm{a}}$. prov. Ap. Gral. 74

I.1 Regla que no fall per haver rao del batiment que lo mestre fa del or portat en la cecca, es aquest: pren l'or fi e alea aquell a la ley que deu esser la moneda que ha batuda, e en aquella aleada aplica lo susteniment que vol lo senyor rey sia mes ultra la ley sua, lo qual es I oz. per desena de marchs aleats, e axi tot multiplicat, aleat e sostengut, ha de aquell or donar rao lo mestre, lo qual or ha esser entre deliurances fetes a ell de moneda e.n obra feta a pes e a ley sua, e en or que comunament li resta en gramalla de la derrera fundicio, e en cisalles de obres, e en minves axi de fondicions com de coloracions.

18 Siguiendo normas recientes de la Comisión Internacional de Diplomática (Folia Cesaraugustana 1, Zaragoza 1984, pp. 75 y ss.) y antiguas costumbres mías, transcribo el texto de acuerdo con las siguientes normas:

a) He retocado ligeramente la presentación del texto para hacerlo más comprensible, alterando, en algunos casos, el orden de los párrafos.

b) Transcribo todas las cifras en arábigo a fin de facilitar su lectura.

c) Mantengo las abreviaturas de marco (m.), onza (oz.), dinero (dr./drs.) y grano (gr./grs.) para los ponderales, y libra (II.), sueldo (ss.) y dinero (d.) para los monetales. En ocasiones, pac. = pacifico.

d) Los párrafos en cursiva están cancelados en el original por líneas que cruzan perpendicular o diagonalmente el texto. Las tachaduras que corresponden a errores de escritura van en nota.

- Tach. florins.

- Tach. al per lo sosteniment a $183 / 4$.

- Signo de llamada.

¿ De otra mano y posterior.

- Tach. que fa.

Tach. dirigida.

8 Tach. a 20 quirats.

h Tach. En aquesta manera que si tor portat en secca seria trobat a 20 quirats, vol que aquell sia pagat per or de 22 quirats, empero comptant aquell del or encamerat que (ilegible).

k Tach. trobat.

1 Tach. encamerat.

$\mathrm{m}$ Tach. de argent a rao de 8.

n Tach. al per la liga. 
1.2 Alear a ley de 20 . Moneda de paciffichs d'or a ley de $20^{\mathrm{b}}$ quirats ab liga de argent pur qui es a ley de 12 diners. Regla.

$5 \mathrm{~m}$. de fi son ................. $6 \mathrm{~m}$. a ley de $20^{\circ}$

$10 \mathrm{~m} . \ldots \ldots \ldots \ldots \ldots \ldots \ldots \ldots \ldots \ldots \ldots \ldots \ldots \ldots \ldots \ldots . .12 \mathrm{~m}$.

$20 \mathrm{~m} . \ldots \ldots \ldots \ldots \ldots \ldots \ldots \ldots . .24 \mathrm{~m}$.

$25 \mathrm{~m} . \ldots \ldots \ldots$ ver $^{\mathrm{d}} \ldots \ldots \ldots \ldots . \quad 30 \mathrm{~m}$.

$50 \mathrm{~m} . \ldots \ldots \ldots \ldots$ ver $^{\mathrm{d}} \ldots \ldots \ldots \ldots .60 .6 \mathrm{~m}$.

$100 \mathrm{~m} . \ldots \ldots \ldots \ldots \ldots \ldots \ldots \ldots \ldots . . .6120 \mathrm{~m}$.

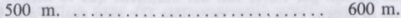

$1000 \mathrm{~m}$. ........................ $1200 \mathrm{~m}$.

1.3 Sustenir. Lo susteniment vol lo senyor rey sia $1 \mathrm{oz}$. per dessena de marchs aleat, ço es 2 parts d'argent e 1 de coure.



I.4 Idem alear a 20 quirats

$5 \mathrm{~m}$. de fi ut supra ........ $6 \mathrm{~m}$. de 20

$1 \mathrm{~m} .2 \mathrm{oz} . \ldots \ldots \ldots \ldots \ldots, 1 \mathrm{~m} .4 \mathrm{oz}$.

- m. 5 oz. (al margen: hic) . - m. $6 \mathrm{oz}$.

- m. 2 oz. 12 drs. ......... - m. $3 \mathrm{oz}$.

$-\mathrm{m} .1 \mathrm{oz} .6 \mathrm{drs} . . . \ldots \ldots,-\mathrm{m} .1 \mathrm{oz} .12 \mathrm{drs}$.

- m. - oz. 15 drs. ......... - m. - oz. 18 drs.

- m. - oz. 7 drs. 12 grs. .. - m. - oz. 9 drs.

- oz. 3 drs. 18 grs. .. - m. - oz. 4 drs. 6 grs.

-oz. - drs. 22 grs. $2 / 4 \quad-$ oz. 1 dr. 3 grs.

- oz. - drs. 11 grs. $1 / 4 \quad-$ oz. - drs. 13 grs. $2 / 4$

-oz. - drs. 5 grs. $2 / 4 \quad-$ oz. - drs. 6 grs. $3 / 4$

- oz. - drs. 5 grs. . $\quad-$ oz. - drs. 6 grs.

Regla que 5 de fi son 6 vulla.s sien m. o oz, o drs. o grs.

I.5 Idem. Susteniment

En $10 \mathrm{~m}$. ut supra $\ldots \ldots \ldots \ldots \ldots, 1 \mathrm{oz}$. qui son a $24 \mathrm{drs}$.

$\mathrm{E}$ axi en $5 \mathrm{~m} . . . \ldots \ldots \ldots \ldots \ldots,-0 z .12 \mathrm{drs}$.

en $2 \mathrm{~m} .4 \mathrm{oz} . \ldots \ldots \ldots \ldots \ldots,-$ oz. 6 drs.

en $1 \mathrm{~m} .2 \mathrm{oz} . \ldots \ldots \ldots \ldots \ldots,-$ oz. 3 drs.

en $-\quad 5$ oz. $\ldots \ldots \ldots \ldots \ldots \ldots-$ oz. 1 dr. 12 grs.

en 2 oz. 12 drs. ....... - oz. - drs. 18 grs.

en $\quad 1 \mathrm{oz}, 6$ drs. $\ldots \ldots \ldots,-$ oz. - drs. 9 grs.

en $\quad-$ oz. 15 drs. ....... - oz. - drs. 4 grs. $2 / 4$

en - oz. 7 drs. 12 grs. ... - drs. 2 grs. $1 / 4$ 


\section{I.6 La pratica}

Summa l'or fi comprat per lo mestre dins

lo temps del present batiment $642 \mathrm{~m} .3 \mathrm{oz} .5 \mathrm{drs} .6 \mathrm{grs} .3 / 4$ Es d'or aleat a ley de paciffichs $770 \mathrm{~m} .7 \mathrm{oz} .1 \mathrm{dr} .12 \mathrm{grs}$. mereyx de sosteniment a rao de $1 \mathrm{oz}$. per desena de marcha aleat $9 \mathrm{~m} .5 \mathrm{oz} .2$ drs. 2 grs. Em axi que monta l'or aleat e sostengut del qual ha dar rao lo mestre ............... $780 \mathrm{~m} .4$ oz. 2 drs. - grs.

Del qual dona rao, primo en deliurances ............. $763 \mathrm{~m} .4 \mathrm{oz} .9$ drs. Item, en or de granalla aleat e sostengut..$\ldots \ldots \ldots \ldots \ldots \ldots$ Item, en cisalla de obres ....... Item, en minves de fondicio .... e en minves de coloracions .... $\begin{array}{ll}-\mathrm{m} . & 2 \mathrm{oz} .11 \mathrm{drs} . \\ -\mathrm{m} . & 5 \mathrm{oz} .19 \mathrm{drs} . \\ 12 \mathrm{~m} . & 2 \mathrm{oz} .7 \mathrm{drs} .1 \mathrm{gr} .3 / 4\end{array}$ $3 \mathrm{~m} . \quad$ oz. 15 grs. 23 (fol. $1 v^{\circ}$ )

II.1 Item, per veure que te del gonyable, pendras les delliuranses fetes a ell en moneda per los moneders, e encara ço que li resta d'or aleat e sostengut de granalla de fondicions e de les cisalles de obres, e ajustat tot lo dit or en suma de marchs, suma que montera a rao de 68 paciffichs d'or de pes per march, e veuras quants paciffichs d'or hi entren en aquella suma d'or que sera delliurat a dit mestre, a feras de aquells paciffichs, lliures a rao de 18 sous paciffich, e vist que sumeran de aquella suma e quantitat leveras lo preu de cost del or fi comprat per lo mestre qui aquell paga semblantment a rao de 18 sous per paciffich, e axi es moneda per moneda.

E la resta ço que sera de lliures, reduyras a lliures de 20 sous paciffich en aquesta forma, que 10 lliures de fot, alias de 18 sous paciffich son 11 lliures de flaca, alias de 20 sous paciffich, segons ja es fet levament posat avant axi de flaca com de fort, e reduyda en dita forma del que montera, levaras les messions que haura fetes lo batiment, e la resta es lo gonyable del senyor rey.

II.2 La pratica

Summa l'or batut delliurat al mestre $763 \mathrm{~m} .4 \mathrm{oz} .9$ drs. Item, en gramalla $2 \mathrm{oz}$. 11 drs. e en cisalla restat al mestre $5 \mathrm{oz}$. 19 drs. 
Es per tot 764 m. 4 oz. 15 drs.

En los quals entren a rao de 68 paciffichs per march ................... 51.990 pac. 17 ss. $91 / 4$ Los quals a rao de 18 sous paciffich valen 56.700 11. 17 ss. 9 d.

De les quals abatudes 40.000

III Vol e axi u ha ordonat lo Senyor duch per sa provisio' dada en Barcelona a 30 de setembre any 1467 que la ley del paciffich sia ${ }^{8}$ de ley de 20 quirats.

Item, ha ordonat que cascun march de paciffichs haia esser de numero de 68 paciffichs d'or, ne mes ne menys, en valor e pes.

Item, ha manat al mestre de la seca que a cascun qui portera or en secca per voler ne fer paciffichs, haja a donar per cascun quirat d'or que sera en lo march 48 sous ${ }^{\mathrm{h}}$, los quals haja a pagar de aquella moneda de paciffichs a rao de 18 sous per cascun paciffich.

(precede al asiento) Del for del or. (de mano posterior)

(al margen) 24 quirats a rao de 2 II. 8 ss. lo quirat val lo march d'or fi 57 ll. 12 ss. raonant paciffich a 18 ss.

Item, que cascun paciffich valga a rao de $18 \mathrm{ss}$. barcelonesos.

Del enantatge dels 2 quirats.

Item, ab altra letra lo dit senyor ha' dada licencia e facultat al mestre de la secca que a qualsevol qui portera or en secca li sia pagat mes avant $\mathbf{2}$ quirats per cascun march ultra la ley en que lo mestre hauria trobat aquell

(precede al asiento) Del enantage (de mano posterior)

(al margen) E mes avant 4 Il. 16 ss. per march de qualsevol ley en que serak $^{k}$ trobat l'or.

\section{IV.1 Or fi}

Val lo march d'or fi portat en secca, a rao de 48 ss. quirat ......... 57 Il. 12 ss.

4 oz. valen ...................... $28 \mathrm{ll} .18$ ss.

2 oz. .......................... 14 Ill. 8 ss.

$1 \mathrm{oz} . \ldots \ldots \ldots \ldots \ldots \ldots \ldots \ldots \ldots \ldots, 7 \mathrm{ll} .4 \mathrm{ss}$.

Valen 12 drs. d'or ................ 3 II. 12 ss.

valen 6 drs. ...................... 1 Il. 16 ss.

valen 3 drs. ...................

valen 2 drs. .....................



valen 12 grs. d'or .................. - Il. 3 ss.

valen 6 grs. ................... - II. 1 ss. $6 \mathrm{~d}$.

valen 3 grs. ...................... - ss. $9 \mathrm{~d}$. 
valen 2 grs. ................... val 1 gr. $\ldots \ldots \ldots \ldots \ldots \ldots \ldots \ldots,-11 .$, ss. $3 \mathrm{~d}$.

IV.2 Enantatge

Val lo march per rao dels 2 quirats del enantatge a la dita rao de 48 ss. quirat ....... 4 Il. 16 ss.

$4 \mathrm{oz}$, valen $\ldots \ldots \ldots \ldots \ldots \ldots \ldots \ldots \ldots, 2 \mathrm{ll} .8 \mathrm{ss}$.

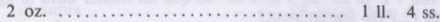

1 oz. .................... 12 ss.

valen 12 drs. d'or $\ldots \ldots \ldots \ldots \ldots \ldots \ldots,-11.6$ ss.

valen 6 drs. .................... 3 ss.

valen 3 drs. ...................

valen 2 drs. .................... 1 ss.



valen 12 grs. d'or ................ - II. - ss. $3 \mathrm{~d}$.

valen 6 grs. .................. II. - ss. $12 / 4$

valen 3 grs. .................... - II. - ss. $1 / 41 / 2$

val 1 gr. $\ldots \ldots \ldots \ldots \ldots \ldots \ldots \ldots \ldots \ldots \ldots \ldots \ldots \ldots$, II. - ss.

IV.3

lo $\mathrm{m}$. del or $\mathrm{fi} \ldots \ldots \ldots \ldots \ldots \ldots \ldots \ldots \ldots \ldots$ Il. 12 ss.

la oz. .......................... 7 II. 4 ss.

lo dr. .................... 6 ss.

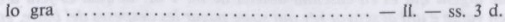

IV.4 Per rao del enantatge dels 2 quirats, ço es

lo $\mathrm{m} . . . \ldots \ldots \ldots \ldots \ldots \ldots \ldots \ldots \ldots . \ldots \ldots$. 4 ll. 16 ss.

la oz. .......................... 12 ss.

lo dr. ..........................

lo gra $\ldots \ldots \ldots \ldots \ldots \ldots \ldots \ldots \ldots \ldots \ldots \ldots \ldots \ldots \ldots \ldots \ldots \ldots$ II. - ss. $1 / 4$

IV.5

Lo mestre paga del fi del or portat a ell en secca verbi gratia es li portat $1 \mathrm{~m}$. d'or de 20 quirats te de fi $6 \mathrm{oz} .16$ drs., valen a rao de $57 \mathrm{ll}$. lo m. del fi ................... $48 \mathrm{II}$.

$\mathrm{E}$ per los 2 quirats del enantage per $\mathrm{m}$. del primer pes e a la ley que.s te obrat de 20 quirats $\ldots \ldots \ldots \ldots \ldots \ldots \ldots \ldots, 4$ Il. 16 ss.

Suma costa or ............... 52 ll. 16 ss. de fort

Les quals $6 \mathrm{oz}$. $16 \mathrm{drs}$. or $\mathrm{fl}$ aleades e sostengudes tornen a ley de 20 quirats que es lo paciffich ................ $1 \mathrm{~m}$.

e lo sosteniment ................ 2 drs. 9 grs. $2 / 4$

valen ...................... 68 pac. II ss. 4 d. de fort valen ......................61 II. 14 ss. $1 \mathrm{~d}$. de fort 
de les quals levades $\ldots \ldots \ldots \ldots \ldots \ldots, 52$ ll. 16 ss.

qui son lo cost del or, resta ........ 8 ll. 18 ss. $1 \mathrm{~d}$. de fort valen de flaca ................. 911.16 ss. $9 \mathrm{~d}$.

de les quals se hauria a pagar mesions de fondre e obrar, e dels altres oficis; la resta es gonyable al senyor rey Es feta estima que les despeses entre carbo, cresols, e fondre e altres officis, loguer de casa e jove son 11.10 ss. $^{e}$ al. 1 ll. 2 ss. $^{e}$ so que ha mester ultra la liga si baixes $(. .$.$) ¿lo sosteniment?$

(fol. $2 v^{\circ}$ )

V.1 Los $25 \mathrm{~m}$. son $30 \mathrm{~m}$. de 20 quirats per ço com los 5 son 6

V.2 Per aleyar $31 \mathrm{~m} .7 \mathrm{oz}$. d'or fi de 24 quirats portat en secca per tornar a la ley de pacifichs de 20 quirats

Los $20 \mathrm{~m}$. de 24 quirats son a 20 quirats ........24 m.

los $10 \mathrm{~m}$............................. $12 \mathrm{~m}$.



$31 \mathrm{~m} .7 \mathrm{oz} . \quad 38 \mathrm{~m} .2 \mathrm{oz}$.

V.3 Aquesta es la regla comuna del aleyar tan solament, empero per pagar les despeses vol lo senyor rey que la toca real sia tan solament de 19 quirats $3 / 4$ de quirat, e per ço es hi ajustat per susteniment 1/4 de argent qui es en cascuna desena de $m$. I oz. la qual es feta d'or en valua e util al senyor rey qui soste pagar les despeses del batre, etc. E per ço hi fa ajustar e de tot deu donar rao lo mestre.

(al margen) Nota. Videretur ut melius

VI.1

En lo m. qui son $8 \mathrm{oz}$. ha haver de

paciffichs d'or ...........68 peces d'or de pes

en $4 \mathrm{oz} . \ldots \ldots \ldots \ldots \ldots \ldots . . \ldots \ldots$ peces

en 2 oz. .............. 17 peces

en $1 \mathrm{oz}$. (al margen) 24 drs... 8 peces 9 ss. de moneda fort de 18 ss. pac.

en 12 drs. d'or (al margen)

mitja oz. ............. 4 peces 4 ss. $6 \mathrm{~d}$. barc. de moneda fort

en 6 drs. ............... 2 peces 2 ss. $3 \mathrm{~d}$.

en 3 drs. ............... 1 peça 1 ss. $1 \mathrm{~d} .2 / 4$ de $\mathrm{d}$.

en 2 drs. .. 12 ss. 8 d. $3 / 4 \ldots$ - peça 9 ss. 3 d. $3 / 4$

en 1 dr. (al margen) 24 grs. 6 ss.

4 d. $1 / 4 \ldots \ldots \ldots \ldots \ldots \ldots$ - peça 4 ss. 9 d. $1 / 4$

en 12 grs. d'or .............. - peça 2 ss. 4 d. $3 / 4$ de d.

$1 / 6$

en 6 grs. ............... - peça 1 ss. 2 d. $1 / 4$ d. 1/3 


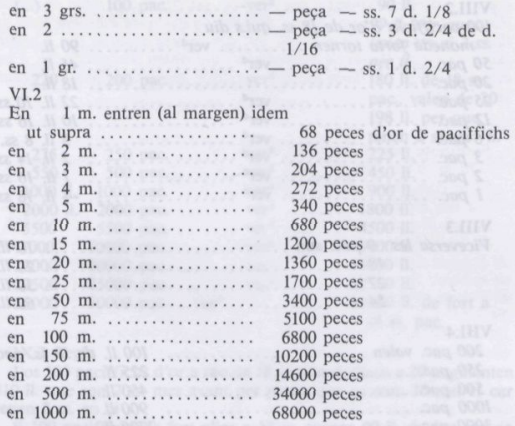

\section{VII.1 Memorial}



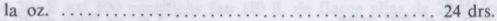

lo dr. $\ldots \ldots \ldots \ldots \ldots \ldots \ldots \ldots \ldots \ldots \ldots \ldots \ldots \ldots \ldots \ldots \ldots, 24$ grs.

VII.2

48 drs. d'or valeri $\ldots \ldots \ldots \ldots \ldots \ldots \ldots \ldots \ldots \ldots, 2 \mathrm{oz}$.

per que $2 \mathrm{~h}$ drs. es ....................... $1 \mathrm{oz}$.

72 drs. .................................. 3 oz.

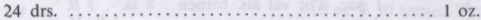

86 drs. ............................

VIII.1

Los 100 pac. d'or de moneda fort a 18 ss. augmenten ...................... II0 Ill. de flaca a $20 \mathrm{~s}$. gui son 2 ss. per pesa los

100 pac, a rao de 18 ss. tornen ...... $90 \mathrm{ll}$. 10 de fort son 11 II. per que tenen 2 ss. en çi

90 pac. de 18 fan de 20 ss. ........ 99 II. de 20 ss.

10 pac. ......................... $11 \mathrm{ll}$.

100 pac. de 18 son ................. I10 II. de 20 
VIII.2

100 paciffichs d'or de 18 ss. qui.s diu

moneda forta tornen ......... ver ${ }^{d}$........ $90 \mathrm{ll}$.

50 pac. .............. ver ${ }^{d} \ldots \ldots \ldots \ldots \ldots .45 \mathrm{ll}$.

20 pac. ................................ $18 \mathrm{ll}$.

25 pac. ................. ver ${ }^{d} \ldots \ldots \ldots \ldots \ldots .22$ II. 10 ss.

12 pac. ................ ver ${ }^{d} \ldots \ldots \ldots \ldots \ldots \ldots, 10$ ll. 16 ss.

6 pac. ............... ver ${ }^{d} \ldots \ldots \ldots \ldots \ldots, 5$ II. 8 ss.

3 pac. ................ ver ${ }^{\mathrm{d}} \ldots \ldots \ldots \ldots \ldots \ldots .2 \mathrm{ll} .14 \mathrm{ss}$.

2 pac. ............... ver ${ }^{d} \ldots \ldots \ldots \ldots \ldots, 1$ Ill. 16 ss.

1 pac. .............. ver ${ }^{d} \ldots \ldots \ldots \ldots \ldots,-11.18$ ss.

\section{VIII.3}

Viceversa les 9 pac. son ..................... $10 \mathrm{ll}$.

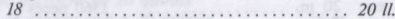

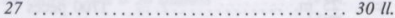

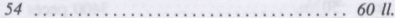

VIII.4

200 pac. valen ................... 100 ll. de 18 ss. pac.

250 pac. ........................ $225 \mathrm{II}$.

500 pac. ...................... $450 \mathrm{ll}$.

1000 pac. ...................... 900 II.

3000 pac. ........................ $2700 \mathrm{ll}$.

5000 pac. ...................... (en blanco)

\section{VIII.5}

4 ss. 6 d. fort valen .............. 5 ss. de flaca de 20 ss.

2 ss. 3 ........................ 2 ss. 6

IX. De moneda fort per reduyr a pagament de moneda flaca (...) 1 pac. d'or val als. tornen . 8 ss. . I II.

(...) $\quad 2$ pac. $\ldots \ldots \ldots \ldots \ldots \ldots \ldots$. 111.16 ss. pac. de 18 ss. $I$ lll. 19 ss. $61 / 4$
(...)
3 pac.
6 pac.
(...) $10 \mathrm{pac}$.
(...) 12 pac.
15 pac.
2 Il. 14 ss. idem.
(...)
(...)
$20 \mathrm{pac}$
25 pac.
5 II. 8 ss. idem.
(...)
50 pac.
911.
10 II. 16 ss. idem.
13 Il. 10 ss. idem.
$18 \mathrm{ss}$.
22 11. 10 ss.
4511.
idem. 49 II. 


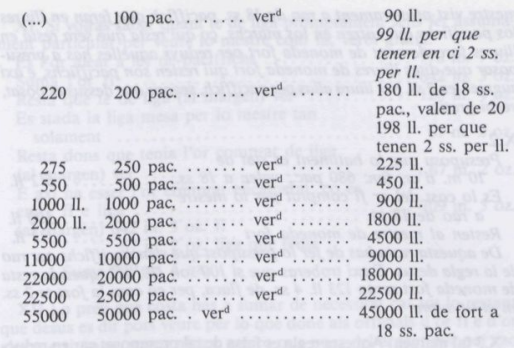

\section{IX.2 Regla}

Los 100 paciffichs d'or a rao de 18 ss. son de flaca a 20 augmenten 110 II. que son 2 ss. mes avant per pessa, per ço com 10 son 11 car tenen 2 ss. en çi

E 100 paciffichs de fort alias a 18 ss. tornen $90 \mathrm{ll}$. de fret per ço com 10 tornen 9

90 paciffichs de 18 fan 99 ll. de 20 per com 10 paciffichs son 1111. de 20 . E axi 100 paciffichs fan 110 Il. de flacca alias de 20 ss. per paciffich.

IX.3 Dels sous de moneda fort

Nota que los 9 son 10 per ço com 18 son 20 , e 20 de fort son 22 de falca per que 10 son 11 per ço com tenen en si 2 sous per paciffich.

18 ss. de moneda fort som ....... 20 ss. de flaca

9 ss. ..................... 10 ss. E 10 tornen 9 vulles pac, d'or, vulles sous en reduccio faedo

4 ss. 6 d. .................. 5 ss.

2 ss. 3 d. .................. 2 ss. $6 \mathrm{~d}$.

1 ss. 1 d. $2 / 4$ de d. ............ 1 ss. $3 \mathrm{~d}$.

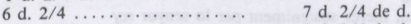

3 d. $1 / 4 \ldots \ldots \ldots \ldots \ldots \ldots .3$ d. $3 / 4$ de $d$.

X.1 Nota que com dels marchs d'or hauras feta reduccio a rao de 68 paciffichs per march en paciffichs d'or e de la suma o quantitat aquella hauras abatuda la quantitat del cost del or fi comprat per lo 
mestre vist primerament a rao de 18 ss. paciffich que feran en lliures los paciffichs qui entren en los marchs, ço qui resta que sera resta en lliures forçadament de moneda fort per reduyr aquelles has a presuposar que dites lliures de moneda fort qui resten son paciffichs, e axi augmenten 2 ss. per lliura alias per paciffich, segons que dessus es posat, verbi gratia.

X.2

Presuposa que lo batiment es stat de

$10 \mathrm{~m}$. d'or, fan: 680 pac.; valen a 18 ss. .......... $612 \mathrm{ll}$.

Es lo cost del or fi comprat per lo mestre

a rao de 18 ss. ........................... $500 \mathrm{ll}$.

Resten al mestre de moneda fort ................ II2 II.

De aquesta resta has de fer lo presupost que sien paciffichs per rao de la regla desus. E axi troberas que si 100 son 110,112 , que.s la resta de moneda fort seran 123 II. 4 ss. de flaca, per ço com la fort te 2 ss. en çi ut supra.

X.3 (al margen) Aquesta regla es falsa de tal presuposat car en reduccio los 9 deven esser 10, altrament seria invers a la cort de 1 per centenar, deus reduyr a altre reduyr. $\mathrm{E}$ axi deu esser que 18 sien 20 . Ideo cancellatum açi, e per lo assumament del marge nota que 1 paciffich de fort no valgue de flach $1 \mathrm{ll}$. $2 \mathrm{ss}$. mas reduynt la resta qui resta pagat l'or no.s deu pendre tal compte ne reduyr que 1 de fort sien 22 ss. de flach, sino que 18 ss. son 20 ss. ut supra.

/(fol. $\left.3 v^{\circ}\right)$

XI.1 Si volras conexer quant ha mester l'or aleat de liga ultra la liga que te en si, es aquesta: pren l'or aleat, e apres de l'aquella suma posa l'or' comprat; la merex mes avant de liga, verbi gratia.

Suma l'or que.s stat aleat a ley de paciffich ................. $770 \mathrm{~m} .7 \mathrm{oz} .12 \mathrm{drs}$.

Es l'or comprat per lo mestre encamerat ............... 709 m. 5 oz. 11 drs. 3 grs.

Resta que merex mes avant de liga que no tenia en si ............. $61 \mathrm{~m} .2$ oz. - drs. 21 grs.

$\mathrm{XI} .2$

Item, merex sosteniment per lo senyor rey a rao de $1 \mathrm{oz}$. per desena de marchs $\mathrm{m}^{\mathrm{m}}$ a ley de 8 drs., ço es 2 parts de argent e 1 de coure ........... $9 \mathrm{~m} .5 \mathrm{oz} .2$ drs. 2 grs.

Es l'or aleat ut supra .......... $770 \mathrm{~m} .7 \mathrm{oz} .12$ drs.

En axi que.s l'or aleat e sostengut .. $780 \mathrm{~m} .4 \mathrm{oz} .18$ drs. 2 
$X I$. Si volras saber quant te de liga promptament sens fer assumament particular per veure lo mestre com fa, verbi gratia.

Es l'or aleat a ley de paciffichs $\ldots \ldots \ldots \ldots \ldots \ldots 770 \mathrm{~m} .7 \mathrm{oz}$.

Suma l'or fi ....................... $642 \mathrm{~m} .3 \mathrm{oz}$.

Resta que te de liga (al margen) ver ${ }^{4} \ldots \ldots \ldots \ldots 128 \mathrm{~m} .4 \mathrm{oz}$.

Es stada la liga mesa per lo mestre tan solament ......................... $61 \mathrm{~m} .2 \mathrm{oz}$.

Resta dons que tenia l'or comprat de liga

(al margen) ver ...................... $67 \mathrm{~m} .2 \mathrm{oz}$.

$\mathrm{E}$ axi ha esser l'or comprat encamerat, ço es

entre fi e liga ......................... $709 \mathrm{~m} .5 \mathrm{oz}$.

(al margen) $642 \mathrm{~m} .3 \mathrm{oz} . \mathrm{fi}$

$$
\text { Suma } \frac{67 \mathrm{~m} .2 \mathrm{oz} . \text { liga que tenia }}{709 \mathrm{~m} .5 \mathrm{oz} .}
$$

XII Lo preu que costa has a sumar de necessitat car tot lo restant que desus es dit pots veure per lo que done als officials d'or fi e d'or aleyat e si no su merex alea e per ço que posa del argent pur per rao del alear ${ }^{n}$ car allo es la liga que lo mestre li done ultra la que te en si l'or comprat. 